\title{
Crop Phenology Estimation using a Multi-temporal Model and a Kalman Filtering Strategy
}

\author{
Fernando Vicente-Guijalba, Tomas Martinez-Marin, and Juan M. Lopez-Sanchez, Senior Member, IEEE
}

\begin{abstract}
In this work a new approach for crop phenology estimation with remote sensing is presented. The proposed methodology is aimed to exploit tools from a dynamical system context. From a temporal sequence of images, a geometrical model is derived which allows us to translate this temporal domain into the estimation problem. The evolution model in state space is obtained through a dimensional reduction by a principal component analysis, defining the state variables, of the observations. Then, estimation is achieved combining the generated model with actual samples in an optimal way using a Kalman filter. As a proof-of-concept, an example with results obtained with this approach over rice fields by exploiting stacks of TerraSAR-X dual polarization images is shown.
\end{abstract}

Keywords-Multi-temporal, SAR, agriculture, phenology, polarimetry, rice, Kalman filter.

\section{INTRODUCTION}

$\mathbf{P}$ RECISION farming is an emerging application of remote sensing for which timely and localized information is essential. In most cases, end-users (i.e. farmers or farm managers) require an accurate knowledge on the status of the crops they are cultivating, since this information leads to trigger or plan different cultivation practices (e.g. irrigation, fertilization, harvest, etc.). The objective of this work is to introduce a novel methodology, based on remote sensing data, aimed to obtain estimations of the current phenological stage of a crop.

Due to its importance as the most widely consumed staple food in the world, rice has been chosen for a first demonstration of this approach. In fact, rice crop monitoring has been a very active subject in remote sensing [1],[2],[3]. As input data we have employed in this work dual polarization synthetic aperture radar (SAR) imagery acquired by the German TerraSAR-X sensor at X-band, which provides a short revisit time (11 days) as required by such an application.

Previous works were focused on obtaining the phenological stage of rice at a given date without using a priori information from previous images or any other auxiliary information [4]. However, current and also future SAR systems with short revisit times, e.g. Sentinel-1 (12 or 6 days) and ALOS-2 (14 days), encourage us to address the phenology estimation problem using a multi-temporal strategy. Recently, diverse multitemporal techniques have been proposed to study the intrinsic

\footnotetext{
This project was supported by the Spanish Ministry of Economy and Competitiveness (MINECO) and EU FEDER under Project TEC2011-28201C02-02. All SAR images have been provided by DLR in the framework of projects LAN0021 and LAN0234 of the prelaunch AO of TerraSAR-X.

The authors are with the Institute for Computing Research, (IUII) University of Alicante, P.O. Box 99, E-03080 Alicante, Spain (e-mail: fernando.vicente@ua.es; tomas@dfists.ua.es; juanma-lopez@ieee.org)
}

dynamics of different land covers as in [5] and [6], and also in [7] where a Kalman filter approach is presented to resolve surface displacement for multi-temporal interferometry. In the literature we can also found monitoring techniques over time series based on data mining as in [8], where an unsupervised approach is employed to extract temporal patterns.

The objective of this work is to establish the phenological stage estimation problem within a dynamical process context which allows us to exploit the tools from the dynamical system analysis in our estimation problem. Although other works in remote sensing are exploiting this same sort of technique, there is still a need to export the dynamical context to the phenology estimation. With this goal in mind, a geometrical model for the phenological evolution is derived from the observed dualpolarimetric data in a set of parcels and employed in combination with observation data to generate an estimation of the phenological stage. It is worth noting that the obtained geometrical model itself contains the intrinsic dynamics of the biophysical process projected into the backscattering radar images. The combination of the derived model and observable data is performed by a Kalman filter in which the statistics from each source are employed to determine the applied gain. As a result, a straightforward methodology for the estimation algorithm is presented, which allows to detect the main phenological stages relevant to the observed backscattering mechanisms. Although this work have been focused on the phenology estimation problem, the model can be employed eventually as a more generic tool, e.g. detection of misbehaviors and diagnosis of pathologies inside fields through signature changes.

In section II the state space representation of the radar observables and also the methodology to obtain the multitemporal evolution model for rice estimation is introduced. Then, in section III the algorithm in the Kalman filter framework used to estimate the field phenology is presented. Results are shown and discussed in section IV. Finally, conclusions are exposed in section $\mathrm{V}$, and some approaches for future work are also pointed out.

\section{Multitemporal MOdel}

\section{A. Rice phenology}

To understand and provide a continuous numerical scale of the plant phenology, the general $\mathrm{BBCH}$ scale for cereals [9] is considered. This classification provides the identification of a number of main stages and subdivides each one into secondary stages. The $\mathrm{BBCH}$ scale also provides a particular representation for rice which is used in this work. In this scale, ten principal growth stages can be identified as shown at the first column of Table I. 
TABLE I. GROWTH STAGES FOR RICE PROPOSED BY THE BBCH SCALE (FIRST COLUMN) AND EQUIVALENT ON RADAR OBSERVATIONS (SECOND COLUMN). DESCRIPTION FOR EACH STAGE IS SHOWN IN THE LAST COLUMN.

\begin{tabular}{ccll}
\hline BBCH Stage & Radar Stage & Description & \\
\hline \hline $0 X$ & 0 & Germination / sprouting & \\
$1 \mathrm{X}$ & $1-2$ & Leaf development & Vegetative \\
$2 \mathrm{X}$ & $2-3$ & Formation of side shoots / tillering & $\begin{array}{l}\text { Phase } \\
3 \mathrm{X}\end{array}$ \\
$4 \mathrm{X}$ & 4 & Stem elongation / rosette growth & \\
\hline $5 \mathrm{X}$ & 5 & Booting & \\
$6 \mathrm{X}$ & 6 & Inflorescence emergence & $\begin{array}{l}\text { Reproductive } \\
\text { Phase }\end{array}$ \\
\hline $7 \mathrm{X}$ & 6 & Flowering & Maturity \\
$8 \mathrm{X}$ & 7 & Development of fruit & Phase \\
$9 \mathrm{X}$ & 7 & Maturity of fruit \& seed & \\
\hline
\end{tabular}

\section{B. Radar observables and dynamical model}

Data provided by a polarimetric SAR sensor, like TerraSAR$\mathrm{X}$, are organized in matrix form by means of the so-called covariance $(\mathrm{C})$ or coherency $(\mathrm{T})$ matrices. From these matrices, it is possible to derive a wide set of polarimetric observables: backscattering coefficients, coherence and phase differences between channels in the linear or Pauli basis, eigenvector and model based decomposition, etc. [10],[11]. With a reduced set of these parameters it is also possible to obtain a coarse estimation of the phenological stage, as it was shown in our previous work [4]. However, in order to minimize the potential loss of information from the original set of radar observations and to provide a continuous phenological stage estimation, we have considered in this study an orthogonal transformation based on the principal component analysis (PCA) [12] over the whole set of dual-polarimetric observables. This transformation allows us to reduce the dimensionality of the observables domain down to just three components. In this way, such transformation enables the definition of a three dimensional state space where the principal components from the observables determines the state variables in the dynamical process.

The input data to generate the dynamical model consists in a set of PolSAR images time series and the correspondent phenologycal ground truth at sufficient temporal rate. The first step is the image coregistration and segmentation of the known rice fields along the time series. One pair of averaged $\mathrm{T}$ and $\mathrm{C}$ matrices are then computed for each parcel and date and decomposed in the commented PolSAR observables. PCA is applied directly over this series of observables which allows us to define a new coordinate system which retains the maximum information in the first three components.

In the defined space it is possible to obtain an evolution of these state variables projecting the average observables to this basis for each parcel. After the projection into this domain, the inherent dynamic signature for each individual parcel becomes relevant. In general, all the rice parcels exhibit a very similar behavior but also preserve their intrinsic details.

Exploiting the signature similarities between fields it is possible to combine, in a local linear least squares sense, the available ground truth and the projected polarimetric observables from a large set of parcels, creating an averaged model at the proposed state space. For the model generation, a set of clusters in the state space domain must be defined by analyzing the individual signatures forming the model. As expected from the rice morphology, the measured radar data do not show the same sensitivity at all $\mathrm{BBCH}$ stages, hence at this point a rearranged classification should be considered to increase the significance of each cluster. In this particular work, eight nonuniform ranges from the original $\mathrm{BBCH}$ scale are considered as clusters to obtain a reliable model. Each cluster defines a phenological stage in the radar domain (shown in the second column in Table I for reference).

As an example of such a model, the average result from a set of six parcels with a dual polarization stack of images over our test site is represented graphically in Figure 1. A significant signature evolution is evident and two regions dominated by an important change in the signature pattern can be recognized. The first one between stages 1 and 2 . At $\mathrm{BBCH}$ 20 plants emergence above the water level occurs resulting in a significant increase in the copolar backscatter responses and a change in the dominant scattering, from surface to doublebounce mechanism.

The second prominent change occurs at stage 4 , on the central vegetative phase development. Throughout this stage dominates a linear decrease in both copolar responses, as a result of the increasing vegetation height and the effect of extinction. Clearly these two stages are driving the internal dynamics of the model. One of the improvements achieved using the proposed methodology with respect to our work in [4] is the potential improvement in the resolution of the phenological estimation. As the model reflects a biophysical behavior, a continuous transition between the modeled stages is expected, and therefore a smooth interpolation allows us to get finer details in the phenological evolution.

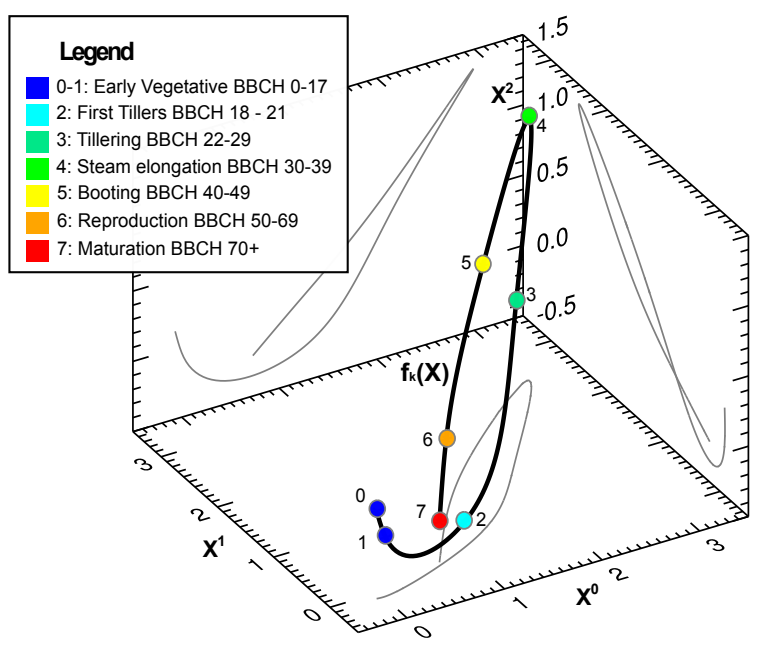

Fig. 1. Average multi-temporal PCA model obtained from the set of radar observables using a set of six rice parcels where phenology is known. Each marked stage represents the cluster center in the state space model.

\section{Statistical characterization}

From the dynamical model it is also possible to obtain a characterization of the covariance matrices at each model 
stage considering the original set of parcel signatures. Thus, each stage $k$ at the model is characterized by its state vector $X_{k}=\left[x_{k}^{0}, x_{k}^{1}, x_{k}^{2}\right]^{T}$ and its associated covariance matrix $Q_{k}$ computed from the set of parcel signatures as in (1).

$$
Q_{k}=E\left\langle\bar{X}_{k} \cdot \bar{X}_{k}^{T}\right\rangle
$$

where $\bar{X}_{k}$ is the normalized state vector at stage $k$. This characterization is employed later inside the estimation-filtering process to determine the model quality at each stage.

\section{PhENOLOGY ESTIMATION}

\section{A. Kalman filter approach}

Using the previously introduced model, an estimation for any field at any date is achieved using a filtering approach. Although estimation can be done employing the model itself, in this work a stronger strategy has been considered and the Kalman filter [13] has been adapted to the phenology estimation framework. For completeness, the main stages on the extended version of the Kalman filter (EKF) [14] approach are shown in Table II.

TABLE II. ESTIMATION STAGES AND DEFINITIONS IN EKF

\begin{tabular}{cl}
\hline Stage & \multicolumn{1}{c}{$\hat{X}_{k \mid k-1}=f_{k-1}\left(\hat{X}_{k-1 \mid k-1}\right)$} \\
\hline \hline Prediction & $P_{k \mid k-1}=J_{k-1} P_{k-1 \mid k-1} J_{k-1}^{T}+Q_{k-1}$ \\
\hline Innovation & \multicolumn{1}{c}{$\tilde{Y}_{k}=Z_{k}-\hat{X}_{k \mid k-1}$} \\
\hline Kalman Gain & $K_{k}=P_{k \mid k-1}\left(P_{k \mid k-1}+R_{k}\right)^{-1}$ \\
\hline & $\hat{X}_{k \mid k}=\hat{X}_{k \mid k-1}+K_{k} \tilde{Y}_{k}$ \\
Update & $P_{k \mid k}=\left(I-K_{k}\right) P_{k \mid k-1}$ \\
\hline Definitions & \\
\hline \hline$\hat{X}_{i \mid j}$, & state vector estimation at $i$ given $j ;$ \\
$f_{i}(X)$, & geometrical model at state variable $X ;$ \\
$J_{i}$, & linearized transition matrix at $i ;$ \\
$Q_{i}$, & model covariance at $i ;$ \\
$P_{i \mid j}$, & prediction covariance at $i$ given $j ;$ \\
$Z_{i}$, & data observation at $i ;$ \\
$\tilde{Y}_{i}$, & innovation at $i ;$ \\
$K_{i}$, & Kalman gain at $i ;$ \\
$R_{i}$, & observation covariance at $i$. \\
\hline
\end{tabular}

In a classical context the filtering process needs a linear analytical expression to define the transition matrix $\left(A_{i}\right)$ which determines how to infer a predicted state from a previous known state $(i)$. Here some considerations have to be taken in order to exploit the expressions from this technique for our purposes. First, to perform an estimation of the phenological stage $k$, the transition matrix $J_{k-1}$ should be computed from the state space model and also linearized in a local neighborhood as an EKF. The linearized transition at state $k-1$ is obtained as the local ratio between joint samples in the model as expressed in (2). This expression allows us to compute the coefficients of the transition matrix relating the model velocity increment between sample models $(k$ and $k-1)$ with the state variable variation in the vicinity of state $k-1$. The obtained transition matrix is related to the Jacobian matrix obtained in case that $f_{k}(X)$ could be expressed as an analytical function:

$$
J_{k-1}^{i j}=\frac{\delta f_{k}^{i}-\delta f_{k-1}^{i}}{X_{k}^{j}-X_{k-1}^{j}}, \quad i, j=0,1,2
$$

In this expression the derivative of the geometrical function at each stage $k$ is computed numerically between interpolated samples as $\delta f=\left(X_{n}-X_{n-1}\right) / \delta t$ in the neighborhood of the current stage.

Once $J_{k-1}$ is known, integration over the curve is accomplished by a numerical integration which allows us to make a prediction for the $n$-th future value based on the previous state variable $k-1$ and the computed transition matrix. As shown in Figure 2, inner state predictions $(n)$ are carried out between acquisitions $(k)$ although the error from the model increases as the integration departs from the local linearization point. There is also a dependency on the nonlinearities within the model and a higher prediction error is expected where a nonlinear behavior is present. In order to obtain reliable estimations the procedure should be performed in two stages, i.e. prediction from the model and update from an observation, as explained in the next section.

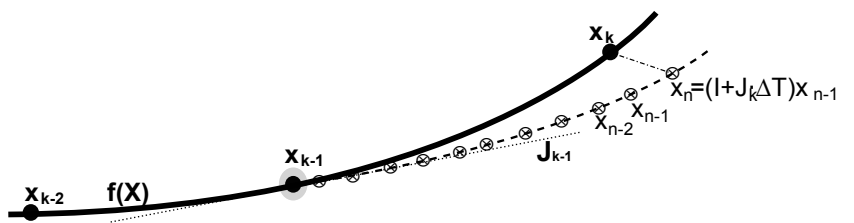

Fig. 2. Geometric model $f(X)$ (continuous line) is linearized in the local neighborhood of the model state $X_{k-1}$. Transition matrix $J_{k-1}$ is employed within a numerical integration to obtain the state predictions $X_{n}$ (dashed line). Integration introduces an accumulative bias from the model.

\section{B. Phenological stage estimation}

As has just been introduced, for each acquisition a prediction is obtained based on the adjusted model and the integrated evolution using the transition matrix $J_{k}$. Here the model is employed to derive a prediction based on the current stage. However, a new image acquisition also produces an observation in the radar domain and a derived $Z_{k}$ in the state space basis which presents an innovation $\tilde{Y}_{k}$ to the predicted value. The covariance matrix for each observation is computed by the Monte-Carlo polarimetric simulation proposed in [10] and [11]. This procedure obtains a distribution of covariance/coherency polarimetric matrices preserving its original statistics. Applying the PCA transformation over the simulated distributions it is possible to derive the observed covariance matrix $R_{k}$ in the state space domain.

Phenology estimation is finally computed as the combination of the predicted state and the observed error difference. The weight of each component in the estimation is set by the Kalman gain $\left(K_{k}\right)$ as expressed in Table II. Moreover, the estimated stage a covariance matrix is also computed, which will be used to feed the system in the next iteration, i.e. when a new acquisition arrives. 


\section{RESULTS}

In this study two stacks of eleven $\mathrm{HH} / \mathrm{VV}$ dual polarization ascending images with a resolution of $6.6 \mathrm{~m}$ and $2.3 \mathrm{~m}$ azimuth and ground range respectively. Each stack was acquired at 30 degrees incidence angle during the 2008 and 2009 cultivation campaigns in a test site located in Sevilla (Spain) where complementary ground truth is available. Details of this dataset can be consulted in [4].

The first step in the estimation process involves the model generation. From a set of parcels where ground truth is available an evolution in the state space domain is obtained. The clustering process over each parcel signature allows us to generate the geometrical evolution model presented in Figure 1. The phenology has to be known in order to perform the clustering process, so the available ground truth constraints the study to a set of six parcels.

Firstly, an example of the results obtained according to the introduced methodology and exploiting the model over one test parcel of 17.5 ha along the 2009 cultivation campaign is shown. Figure 3 presents the comparison between the supplied ground truth and the mean and standard deviation of the phenology estimates at each acquisition. Due to the cluster computation, the first stage in the model is centered around $\mathrm{BBCH} 15$, hence a lack of sensitivity in the geometrical model is observed for the very first acquisition and an overestimation, due to a saturation effect, is obtained. From here and until the last stage a good agreement is obtained between ground truth and the average estimation, although an slight overestimation is present. This behavior was expected due to the way the ground truth is provided: the $\mathrm{BBCH}$ stage provided for a parcel is that value which more than the $50 \%$ of the parcel has reached. For the final stages an increase in the estimation variance is present. This is explained by the high variability present in the radar data during the latest development stages, as observed in [4]. Moreover, it is possible to observe in the dynamical model, shown in Figure 1, the proximity of stages 2 and 7. This closeness agrees with the results obtained in [4], where the phenology estimates in areas actually belonging to the maturation stage where wrongly retrieved as in earlier stages.

In Figure 4 the spatial distribution of phenology estimates obtained for the test parcel is shown. As a reference, the supplied ground truth for each acquisition is also included in the ground truth row. As pointed out before, the available ground truth consists in a single phenology data for each date at a single parcel, preventing us from performing a comparison at the pixel level.

The heterogeneities detected inside the fields should be compared with higher resolution ground truth data in order to validate further this methodology.

For the purpose of comparing the obtained results with those obtained with the procedure proposed in [4], which only makes use of a single radar acquisition to estimate phenology, hereafter called static methodology, Figure 5 shows the estimation results for both approaches coded with the same five-stages estimation scale used in the previous study. With the static approach there are some pixels, falling outside the rules of the algorithm, which result in unassigned to any stage, and

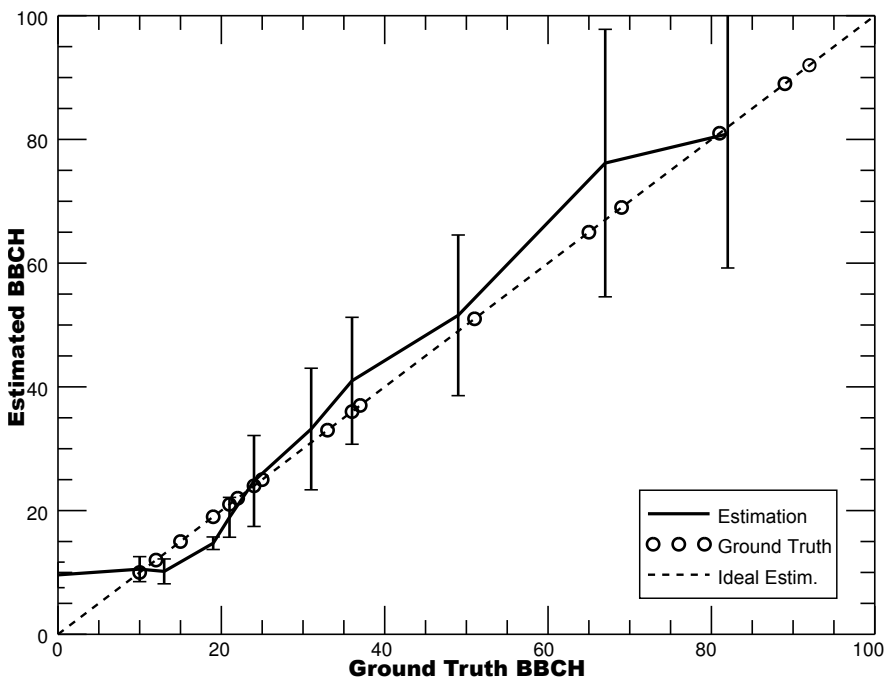

Fig. 3. Comparison of ground truth versus estimation results in the 2009 cultivation campaign. Dashed line (1:1 line) indicates the ideal estimation and circles the provided ground truth for the test parcel. Continuous line corresponds to the interpolated mean estimation results. Deviation inside parcel for each acquisition marked with whiskers.

their number increases at the latest acquisitions. This effect is directly avoided by the dynamical approach, since estimation is obtained for every pixel in the scene. In general, results from the dynamical estimation produces homogeneous phenological values for almost all acquisitions. For instance, at DoY 170 noticeable differences are obtained between both methods. The unreliable extreme values obtained with the static method are not present in the dynamical result, although some delayed evolutions are still present. On the contrary, a good agreement exists in the next acquisition (DoY 181) where the visible heterogeneities were confirmed by personnel of the ground campaign. Despite the coverage inside the field is higher in the dynamical estimation technique, it is evident that there is an accumulative effect in the estimation process which has its impact directly into the variability of the estimation results.

\section{CONCLUSION AND DISCUSSION}

In this work we have proposed and tested a multi-temporal dynamical approach for crop phenological stage estimation using remote sensing imagery. The main contribution in this study is the definition of a new strategy for the generation of a dynamical model which allows us to move into the state space domain. Although this work has given a specific solution for rice using a set of SAR images the general approach can be applicable to any remote sensing data. This methodology also sets up the basis to integrate different sources of information into the geometrical evolution model. For instance, integration derived from the data fusion of a multi-track set of SAR images or from ascending or descending orbits. Different incidence angles could be considered too, aimed to increase the temporal resolution of both the model and the estimates. Furthermore, the methodology is able to incorporate any other type of auxiliary information if available. 


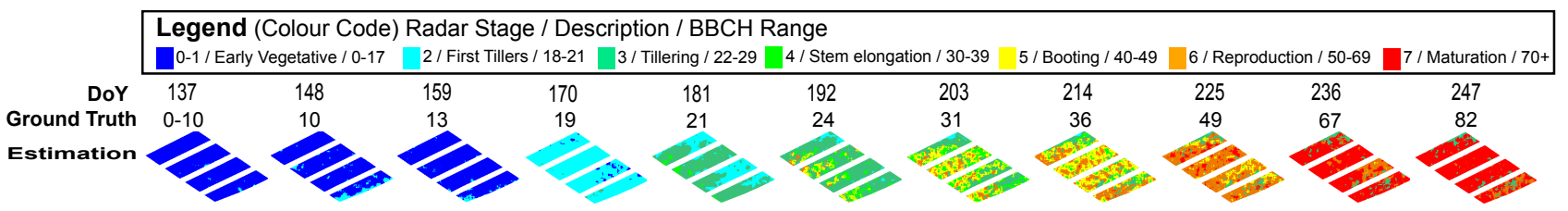

Fig. 4. Phenological estimation results obtained over the test parcel at pixel basis with the proposed methodology. Temporal scale in Days of Year (DoY) and ground truth in $\mathrm{BBCH}$ scale.

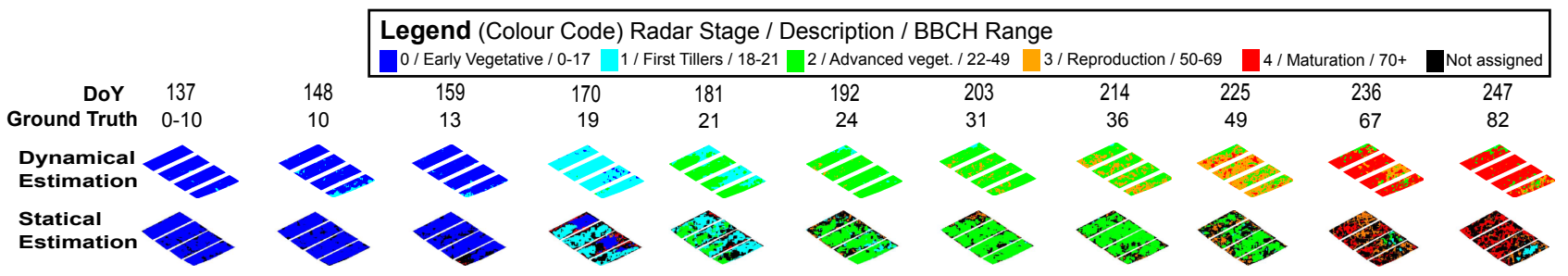

Fig. 5. Results from dynamical (top row) and statical (bottom row) estimation at test parcel. Each set of results corresponds to the test field within the 2009 cultivation campaign although the mask layout is slightly restrictive in the dynamical case.

For the estimation problem, the contribution of this study lies in the adaption of a conventional Kalman filter approach. The linearized version of the transition matrix allows us to understand the estimation in a classical way. However, a deeper analysis should be conducted to detect how this linearization affects in the proximity of nonlinearities inside the model and, consequently, to make an effort to avoid this. In this sense, in future works an advanced filtering strategy could be considered, using Particle Filtering techniques. Although some other aspects should be considered in a final application, this technique has been proven to provide good estimations on rice fields based on dual-pol SAR imagery.

Ongoing work is addressing to derive a model for another types of crops, which may present similar signatures at the proposed state space basis. Also the model derivation with a more complete campaign, even with data in controlled environments, would allow us to determine the full capabilities of this procedure.

\section{ACKNOWLEDGMENT}

The authors would like to thank the Federacion de Arroceros de Sevilla for providing the ground measurement data.

\section{REFERENCES}

[1] T. Kurosu, M. Fujita, and K. Chiba, "Monitoring of rice crop growth from space using the ERS-1 C-band SAR," IEEE Trans. on Geoscience and Remote Sensing, vol. 33, pp. 1092-1096, 1995.

[2] T. Le Toan, F. Ribbes, L.-F. Wang, N. Floury, K.-H. Ding, J.A. Kong, M. Fujita, and T. Kurosu, "Rice crop mapping and monitoring using ERS-1 data based on experiment and modeling results," IEEE Trans. on Geoscience and Remote Sensing, vol. 35, pp. 41-56, 1997.

[3] A. Bouvet, T. Le Toan, and N. Lam-Dao, "Monitoring of the rice cropping system in the Mekong delta using ENVISAT/ASAR dual polarization data," IEEE Trans. on Geoscience and Remote Sensing, vol. 47, pp. 517-526, 2009.

[4] J. M. Lopez-Sanchez, S. R. Cloude, and J. D. Ballester, "Rice phenology monitoring by means of SAR polarimetry at X-band," IEEE Trans. on Geoscience and Remote Sensing, vol. 50, no. 7, pp. 2695-2709, July 2012
[5] P. Marzahn, K. Kruger, and R. Ludwig, "Derivation of soil surface roughness dynamics from multi-temporal and multi-parametric airborne PolSAR-data," in Analysis of Multi-temporal Remote Sensing Images, 2007. MultiTemp 2007. International Workshop on the, 2007, pp. $1-5$.

[6] C. Marechal, E. Pottier, S. Allain-Bailhache, S. Meric, L. Hubert-Moy, and S. Corgne, "Mapping dynamic wetland processes with a one year RADARSAT-2 quad pol time-series," in Geoscience and Remote Sensing Symposium (IGARSS), 2011 IEEE International, 2011, pp. 126129.

[7] J. Hu, X.-L. Ding, Z.-W. Li, J.-J. Zhu, Q. Sun, and L. Zhang, "Kalmanfilter-based approach for multisensor, multitrack, and multitemporal InSAR," IEEE Trans. on Geoscience and Remote Sensing, 2013, [In press], DOI: 10.1109/TGRS.2012.2227759.

[8] A. Julea, N. Méger, C. Rigotti, E. Trouvé, P. Bolon, and V. Lăzărescu, "Mining pixel evolutions in satellite image time series for agricultural monitoring," in Proc. of the 11th international conference on Advances in data mining: applications and theoretical aspects. 2011, ICDM'11, pp. 189-203, Springer-Verlag.

[9] U. Meier, "Growth stages of mono-and dicotyledonous plants (2nd ed.)," Tech. Rep., Federal Biological Research Centre for Agriculture and Forestry, 2001.

[10] S. R. Cloude, Polarisation. Applications in remote sensing, Oxford University Press, 2009.

[11] J.-S. Lee and E. Pottier, Polarimetric radar imaging. From basics to applications, CRC Press, 2009.

[12] L. Eklundh and A. Singh, "A comparative analysis of standardised and unstandardised principal components analysis in remote sensing," International Journal of Remote Sensing, vol. 14, no. 7, pp. 1359-1370, 1993.

[13] R. E. Kalman, "A new approach to linear filtering and prediction problems," Transactions of the ASME Journal of Basic Engineering, vol. 82, pp. 35-45, 1960.

[14] R. E. Kalman and R. S. Bucy, "New results in linear filtering and prediction theory," Transactions of the ASME Journal of Basic Engineering, vol. 83, pp. 95-107, 1961. 